\title{
Spatio-temporal Functional Regression on Paleo-ecological Data
}

\author{
Liliane Bel ${ }^{*}$, \\ UMR 518 AgroParisTech/INRA,16, rue Claude Bernard - 75231 Paris Cedex 05 \\ Avner Bar-Hen, \\ Université René Descartes, MAP5, 45 rue des Saints Pères, 75270 Paris cedex 06 \\ Rachid Cheddadi, \\ ISEM, case postale 61, CNRS UMR 5554, 34095 Montpellier, France \\ Rémy Petit, \\ UMR 1202 INRA , 69 route d'Arcachon 33612 Cestas Cedex, France
}

\begin{abstract}
The influence of climate on biodiversity is an important ecological question. Various theories try to link climate change to allelic richness and therefore to predict the impact of global warming on genetic diversity. We model the relationship between genetic diversity in the European beech forests and curves of temperature and precipitation reconstructed from pollen databases. Our model links the genetic measure to the climate curves through a linear functional regression. The interaction in climate variables is assumed to be bilinear. Since the data are georeferenced, our methodology accounts for the spatial dependence among the observations. The practical issues of these extensions are discussed.
\end{abstract}

Key words: Functional Data Analysis; Spatio-temporal modeling; Climate change; Biodiversity 


\section{Introduction}

2 Climate records show that the earth has recorded a succession of periods of

* Corresponding author.

Email address: Liliane.Bel@agroparistech.fr (Liliane Bel). 
1

\section{Data}

and concluding remarks are given in Section 5 .

\section{.}

Pollen records are important proxies for the reconstruction of climate parameters since variations in the pollen assemblages mainly respond to climate changes. Based on the fossil and surface pollen data from pollen databases, we used modern analogue technique (MAT) to reconstruct climate variables. Climate reconstruction is accomplished by matching fossil biological assemblages to recently deposited (modern) pollen assemblages for which climate properties are known. The relatedness of fossil and modern assemblages is usually measured using a distance metric that rescales multidimensional species assemblages into a single measure of dissimilarity. The distance-metric method is widely used among paleoecologists and paleoceanographers [8]. Temperature and precipitation were reconstructed at 216 locations from the present back to a variable date depending on available data. The pollen dataset was used to reconstruct climate variables, throughout Europe for the last 15000 years of the Quaternary. Due to the methodology, each climate curve is sampled at irregular times for each location.

Genetic diversities were measured from variation at 12 polymorphic isozyme loci in European beech (Fagus sylvatica L.) forests based on an extensive sample of 389 populations distributed throughout the species range. Based on these data, various indices of diversity can be computed. They mainly characterize within or between population diversity. In this article, we focus on the $H$ index, the probability that two alleles sampled at random are different. This parameter is a good indication of gene diversity [3].

The two datasets were collected independently and their locations do not coincide. 


\section{Functional Regression}

2 The functional linear regression model with functional or real response has

3 been the focus of various investigations [1, 6, 67, 11]. We want to estimate the

${ }_{4}$ link between the real random response $y_{i}=d\left(s_{i}\right)$, the diversity at site $s_{i}$ and

${ }_{5}\left(\theta_{i}(t), \pi_{i}(t)\right)_{t>0}$ the temperature and precipitation functions at site $s_{i}$. There

6 are two points to consider for the modeling: (i) functional linear models need

7 to be extended to incorporate interaction between climate functions; (ii) since

8 we have spatial data, observations cannot be considered as independent and

9 we also need to extend functional modeling to account for spatial correlation.

We assume that the temperature and precipitation functions are square integrable random functions defined on some real compact set $[0, T]$. The very general model can be written as:

$$
Y=f\left((\theta(t), \pi(t))_{T>t>0}\right)+\varepsilon
$$

10 $f$ is an unknown functional from $L^{2}([0, T]) \times L^{2}([0, T])$ to $\mathbb{R}$ and $\varepsilon$ is a spatial

11

12

13

14

$15 \quad A$ and $B$ are in $L^{2}([0, T])$ and $C$ is a kernel of $L^{2}([0, T])$.

Let $\left(e_{k}\right)_{k>0}$ be an orthonormal basis of $L^{2}([0, T])$. Expanding all functions on this basis we get

$$
\theta_{i}(t)=\sum_{k=1}^{+\infty} \alpha_{k}^{i} e_{k}(t) \quad \pi_{i}(t)=\sum_{k=1}^{+\infty} \beta_{k}^{i} e_{k}(t)
$$




$$
A(t)=\sum_{k=1}^{+\infty} a_{k} e_{k}(t) \quad B(t)=\sum_{k=1}^{+\infty} b_{k} e_{k}(t) \quad C(t, u)=\sum_{k, \ell=1}^{+\infty} c_{k \ell} e_{k}(t) e_{\ell}(u)
$$

and

$$
y_{i}=\mu+\sum_{k=1}^{+\infty} a_{k} \alpha_{k}^{i}+\sum_{k=1}^{+\infty} b_{k} \beta_{k}^{i}+\sum_{k, \ell=1}^{+\infty} c_{k \ell} \alpha_{k}^{i} \beta_{\ell}^{i}+\varepsilon_{i}
$$

1

$$
\begin{aligned}
& X=\left(\begin{array}{ccccc}
\alpha_{1}^{1} \ldots & \alpha_{K}^{1} \beta_{1}^{1} \ldots & \beta_{K}^{1} & \alpha_{1}^{1} \beta_{1}^{1} \ldots & \alpha_{K}^{1} \beta_{K}^{1} \\
\vdots & \ldots & \vdots \\
\alpha_{1}^{n} \ldots & \alpha_{K}^{n} \beta_{1}^{n} \ldots & \beta_{K}^{n} & \alpha_{1}^{n} \beta_{1}^{n} \ldots & \alpha_{K}^{n} \beta_{K}^{n}
\end{array}\right) \quad \operatorname{dim}(X)=n \times\left(2 K+K^{2}\right) \\
& \operatorname{cov}\left(\varepsilon_{i}, \varepsilon_{j}\right)=\rho\left(s_{i}-s_{j}\right)
\end{aligned}
$$

$$
\sqrt{n}(\widehat{\phi}-\phi) \rightarrow \mathcal{N}\left(0, \lim _{n \rightarrow \infty} n\left(X^{t} \Sigma^{-1} X\right)^{-1}\right)
$$

10 The estimation of $\Sigma$ is convergent under mild conditions [4] and the conver-

In order to estimate the regression and the correlation function parameters we proceed by Quasi Generalized Least Squares: a preliminary estimation of $\phi$ is given by Ordinary Least Squares, $\phi^{*}=\left(X^{t} X\right)^{-1} X^{t} Y$, the correlation function is estimated from the residuals $\widehat{\varepsilon}=Y-X \phi^{*}$ and the final estimate of $\phi$ is given by plugging the estimated correlation matrix $\widehat{\Sigma}$ in the Generalized Least Squares formula $\widehat{\phi}=\left(X^{t} \widehat{\Sigma}^{-1} X\right)^{-1} X^{t} \widehat{\Sigma}^{-1} Y$. If both estimations of $\phi$ and $\Sigma$ are convergent and assuming normal distribution of the residuals then [9]:

gence of $\phi$ is assessed for example when the functions are expanded on a splines basis [1] or on a Karhunen expansion [10].

Significance of the predictors can be tested if the residuals are assumed to be Gaussian, within the classical framework of linear regression models.

Several parameters need to be set. The first choice is that of the orthonormal 
1 basis. It can be Fourier, splines, orthogonal polynomials, wavelets. Then the 2 order of truncation has to be determined. The spatial correlation function 3 of the residuals may be of parametric form (exponential, Gaussian, spherical 4 etc.). These choices will be made by minimizing a cross validation criterion: a 5 sample with no missing data for all variables is determined, and for each site of 6 the sample a prediction of the diversity is computed according to parameters 7 estimated without the site in the sample. The global criterion is the quadratic 8 mean of the prediction error.

\section{Results}

Pollen was collected throughout Europe providing temporal estimation of temperatures and precipitation. These estimations are not regularly spaced, and have very different ranges from 1 Kyears to 15 Kyears. Beech genetic indices are recorded in forests and do not coincide with the pollen locations. Figure 1 shows the locations of the two datasets.

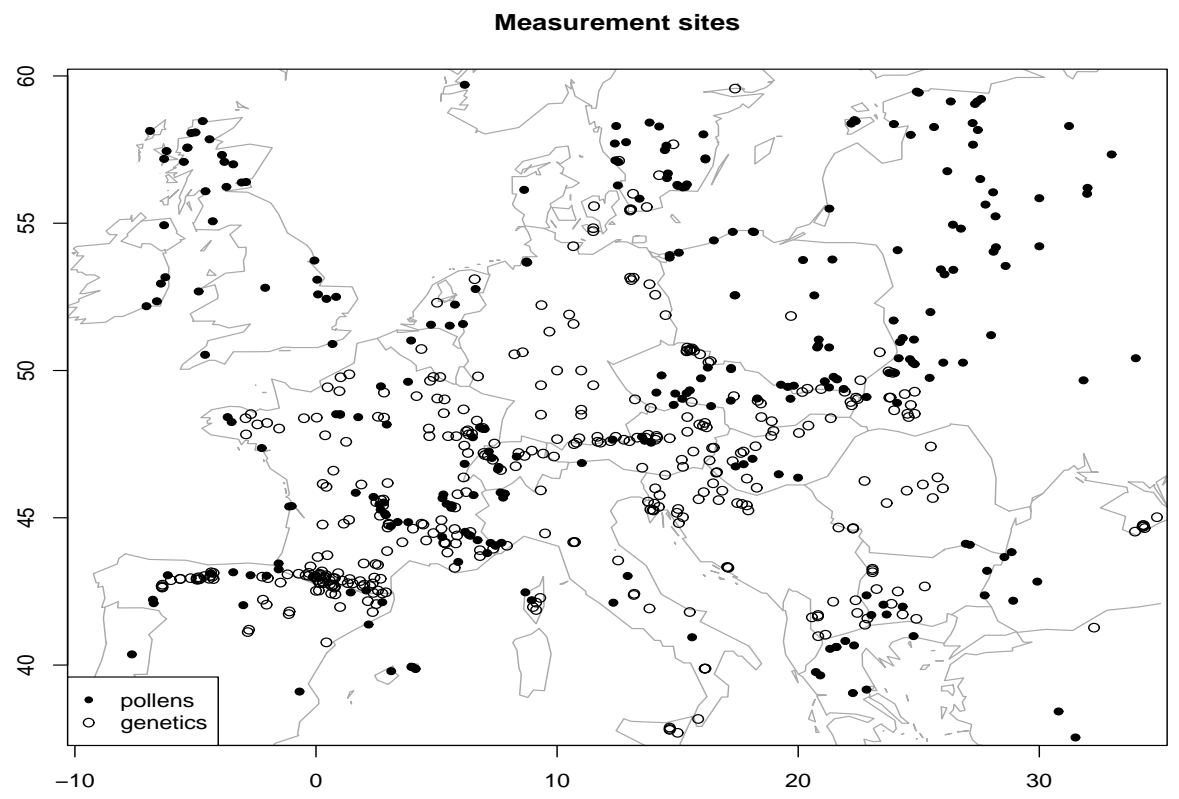

Figure 1. Locations of pollen (black dots) and genetic (open circles) records. 
1 Climate variables are continuous all over Europe but beech forests have specific 2 locations. In order to make our data to spatially coincide, temperature and 3 precipitation curves are firstly estimated on a regular grid of time from 15 4 Kyears to present on sites where are collected the genetic measures. 15 Kyears 5 corresponds to the beginning of migration of plants onto areas made free by 6 the retreating ice sheets.

7 The interpolation is done by a spatio-temporal kriging assuming the covariance function is exponential and separable. Figure 2 shows for a particular site the estimated temperature curve together with some neighboring curves issued from collected pollen.

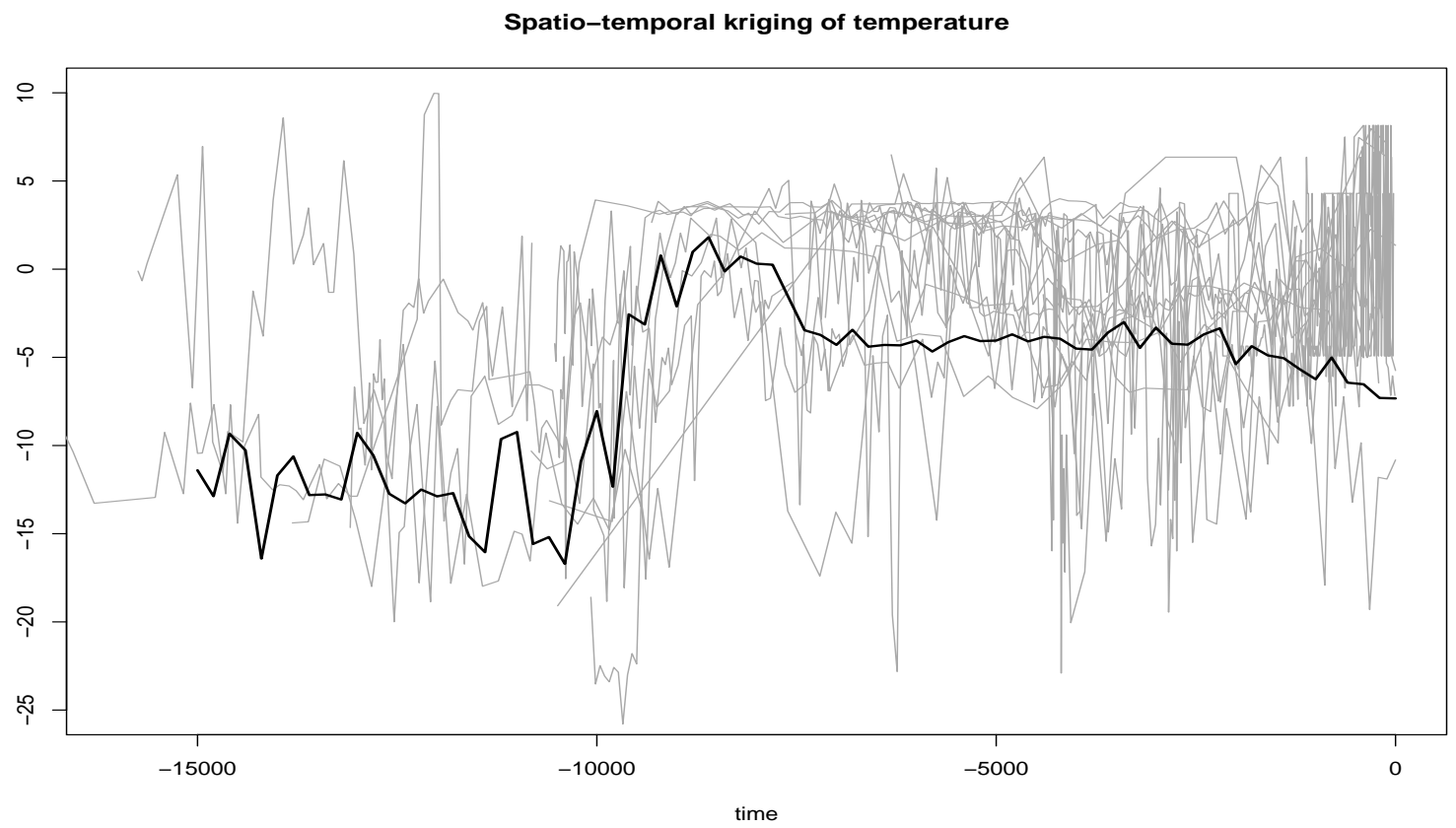

Figure 2. Resulting temperature curve (thick black curve) from spatio-temporal kriging of 20 neighboring temperatures curves from recorded pollen.

We aim to predict genetic diversity with precipitation and temperature curves.

This corresponds to a functional regression model with genetic diversity as dependent variable and temperature and precipitation curves as predictor variable. The cross validation criterion gives better results with an expansion of the predictor variables on a Fourier basis of order 5 . Figures 3 and 4 show the coefficient functions $A, B$, and kernel $C$. 

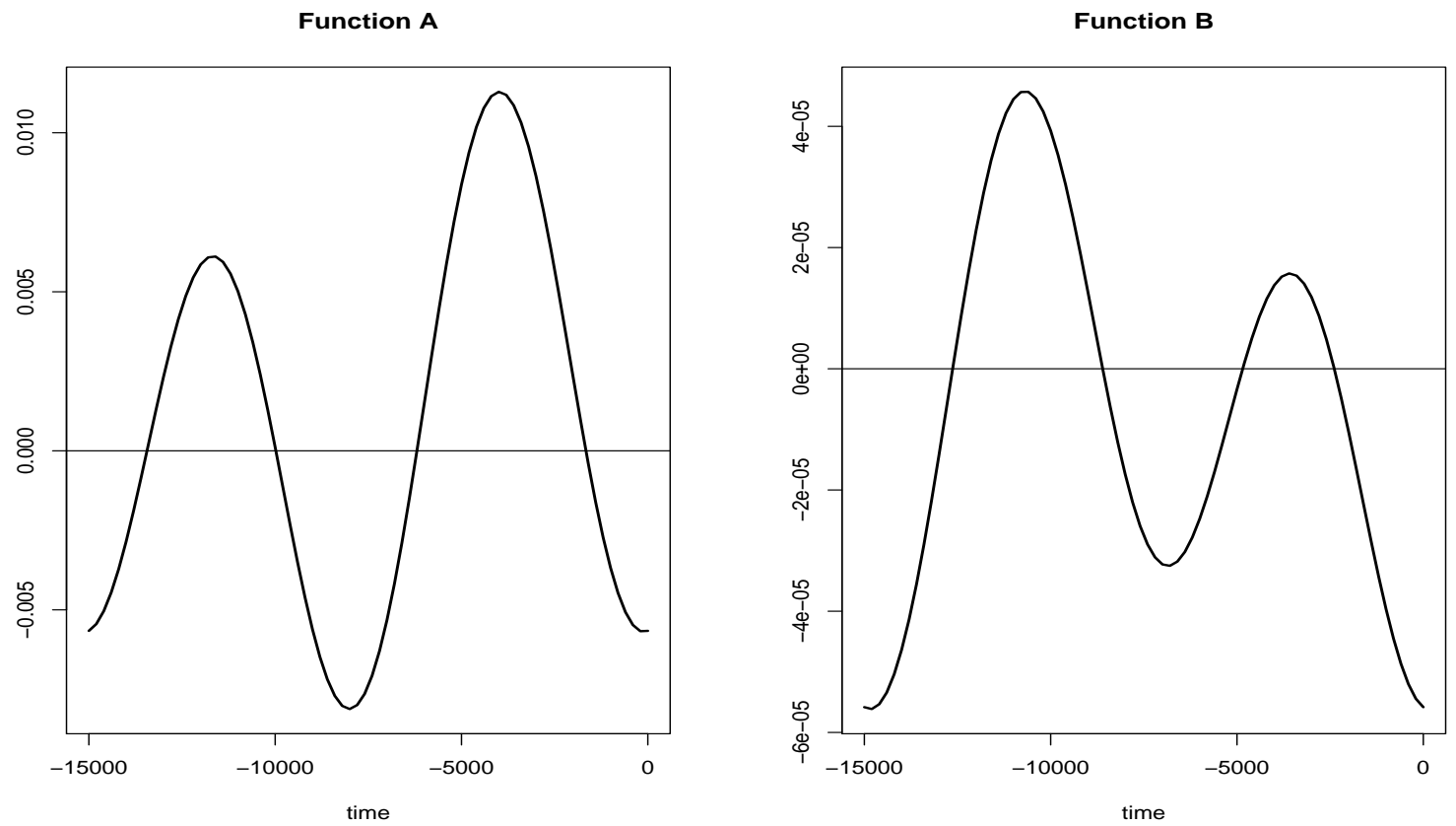

Figure 3. Coefficient function $A$ of the temperature and coefficient function $B$ of the precipitation

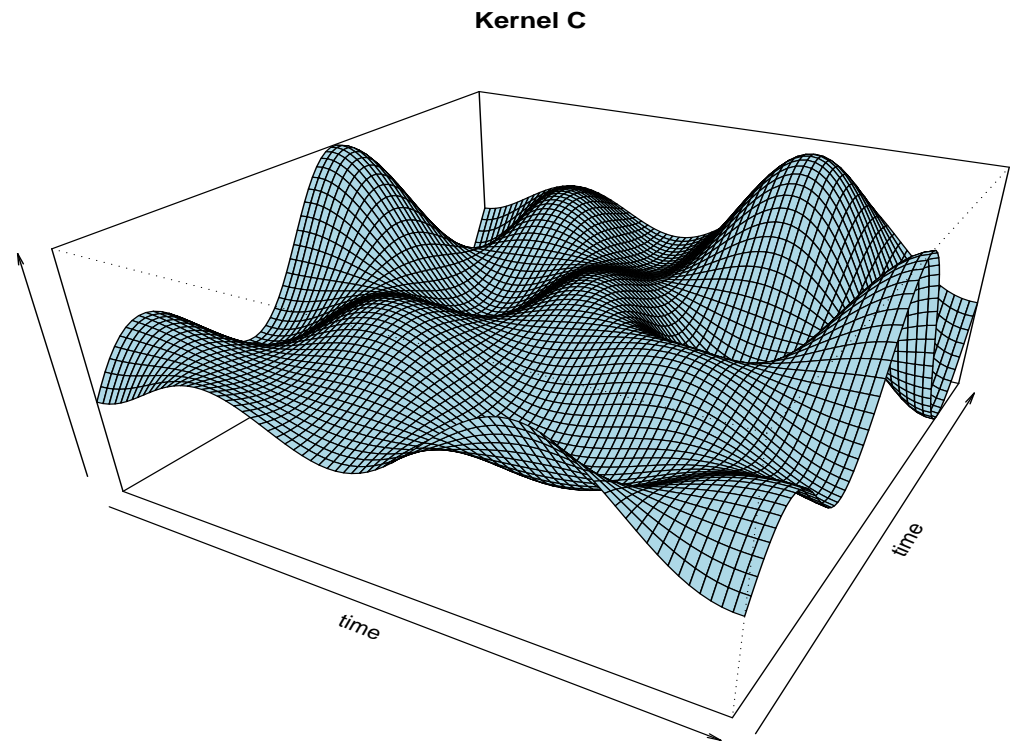

Figure 4. Kernel $C$ of the interaction temperature-precipitation

1 The shape of the coefficient function $A$ shows that the term $\langle A, \theta\rangle$ will be 2 higher when the gap between periods before 7.5 Kyears and after 7.5 Kyears 
1 is higher (temperatures before 7.5 Kyears are mostly negative), meanwhile the 2 shape of the coefficient function $B$ shows that the term $\langle B, \pi\rangle$ will be higher 3 when the precipitation before 7.5 Kyears is higher (precipitation is positive).

${ }_{4}$ The surface of kernel $C$ is obviously not the product of two curves in the two 5 coordinates, showing an effect of interaction.

6 In Figure 5 the residual variogram graph exhibits some spatial dependence. An 7 exponential variogram is fitted, and the resulting covariance matrix is plugged 8 into the GLS formula to update the coefficients and test the effects of the 9 temperature, precipitation and interaction.

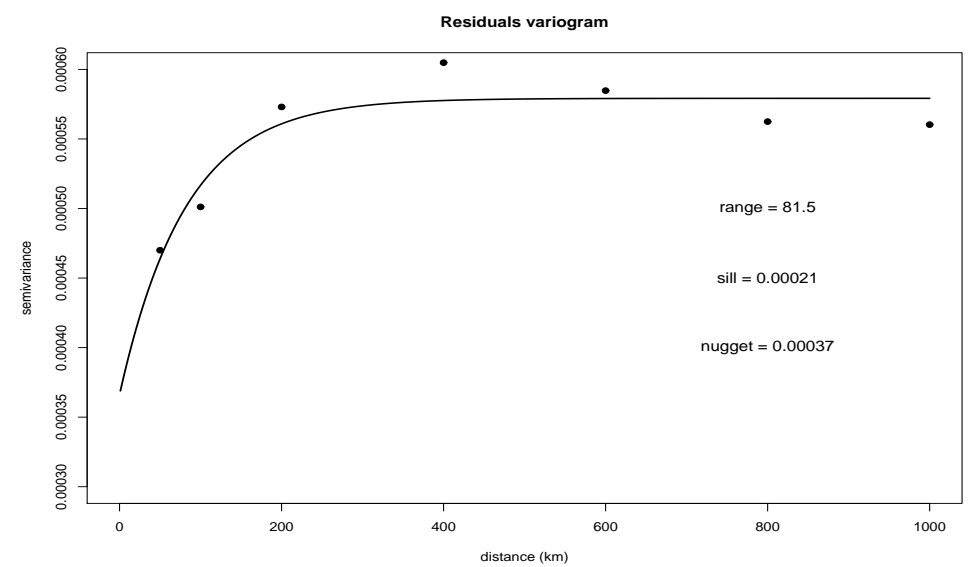

Figure 5. Empirical and fitted variogram on the residuals.

10 The graphs in Figure 6 show that the model explains a part of the diversity 11 variability. However it is far from explaining all the variability as the $R^{2}$ is 12 equal to 0.31 . 

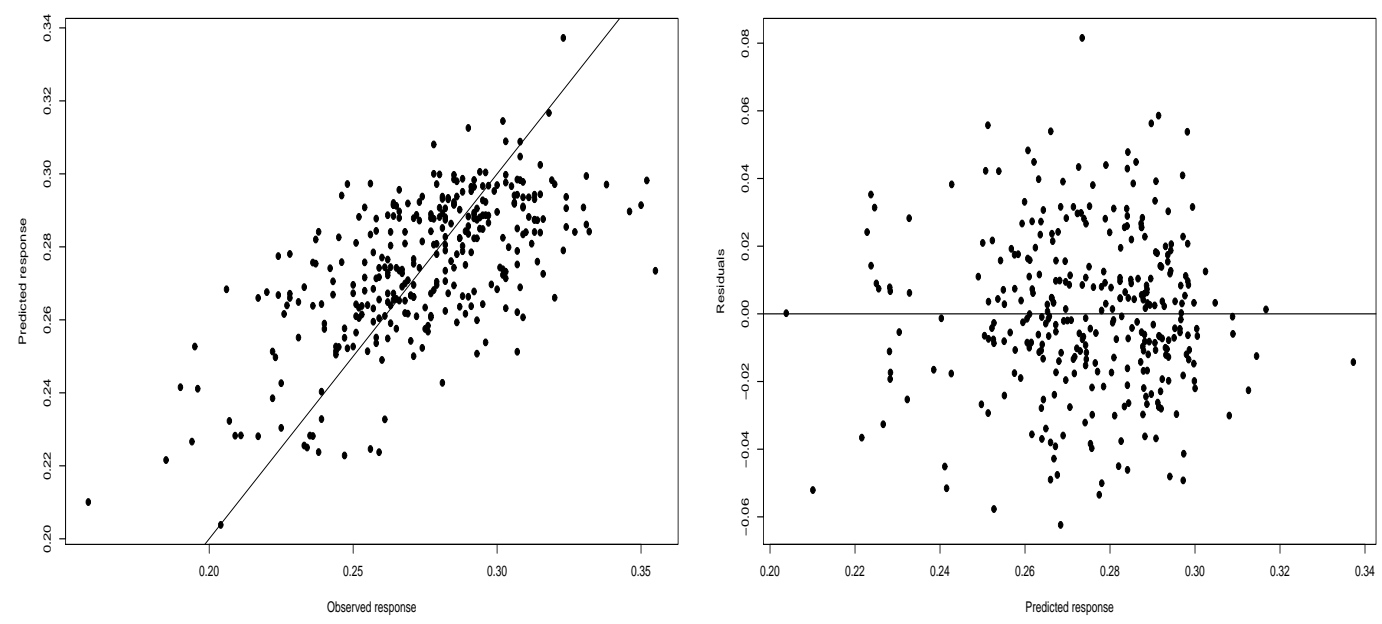

Figure 6. Observed-Predicted response and Predicted-Residuals graphs.

1 Table 1 gives the analysis of variance of the four nested models:

2 Model 1: $\mathbb{E}(Y)=\mu+\langle A ; \theta\rangle+\langle B ; \pi\rangle+\langle C \theta ; \pi\rangle$

3 Model 2: $\mathbb{E}(Y)=\mu+\langle A ; \theta\rangle+\langle B ; \pi\rangle$

${ }_{4} \quad$ Model 3: $\mathbb{E}(Y)=\mu+\langle A ; \theta\rangle$

${ }_{5}$ Model 4: $\mathbb{E}(Y)=\mu+\langle B ; \pi\rangle$

Table 1

Analysis of variance models of nested models

6 The $p$-values $(2.2 \mathrm{e}-16)$ of the tests $\mathrm{H}_{0}$ : model 3 (model 4 ) against $\mathrm{H}_{1}$ : model

72 and $(1.430 \mathrm{e}-07)$ of the test $\mathrm{H}_{0}$ : model 2 against $\mathrm{H}_{1}$ : model 1 show that the 8 interaction and the two variables have a strong effect. 
Temperature
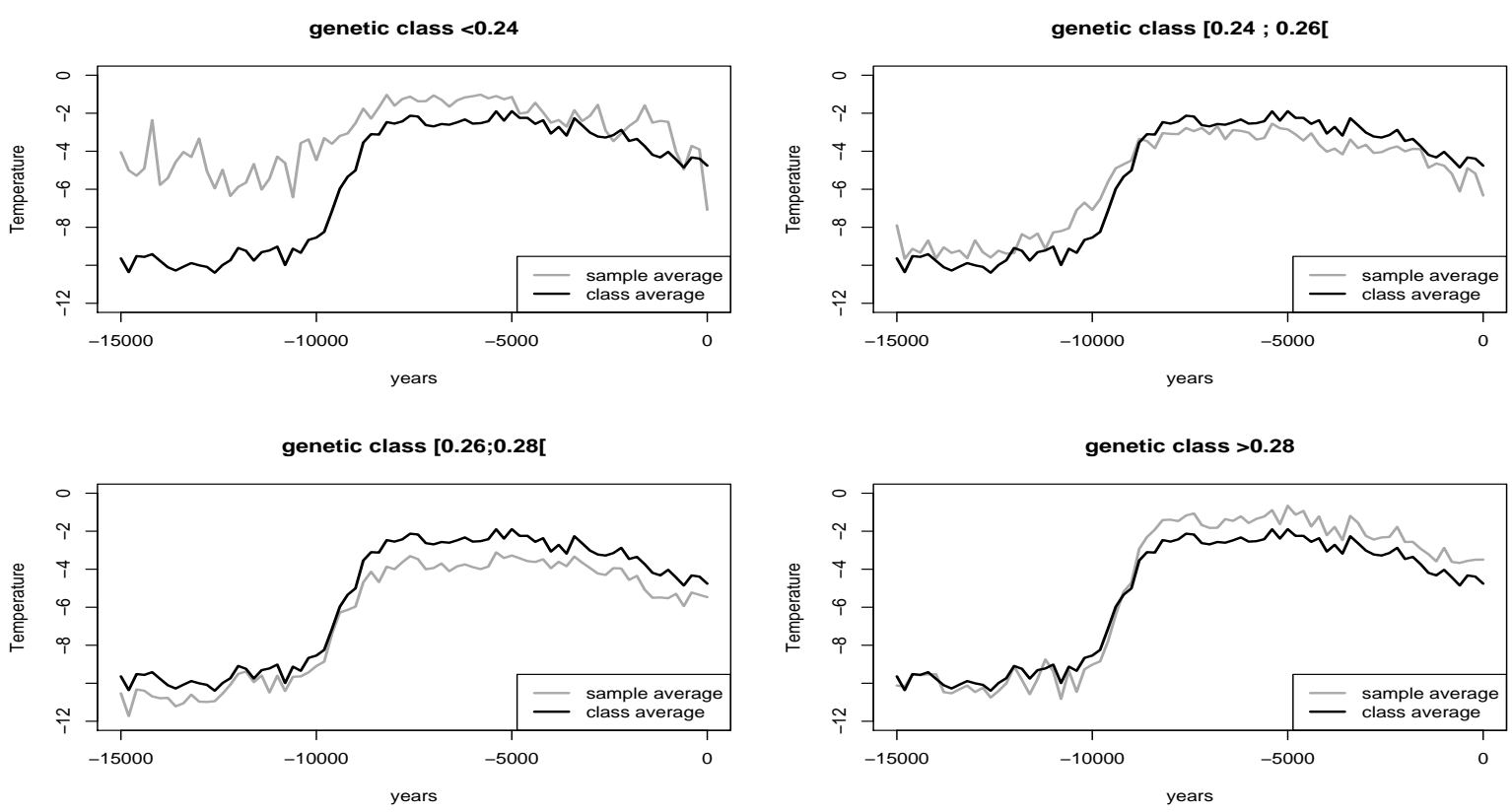

Figure 7. Pattern of temperature curves according to the range of the predicted response.

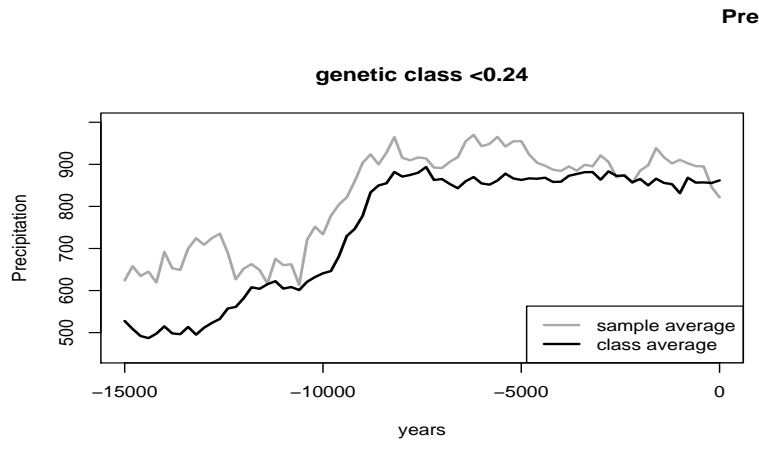

recipitation
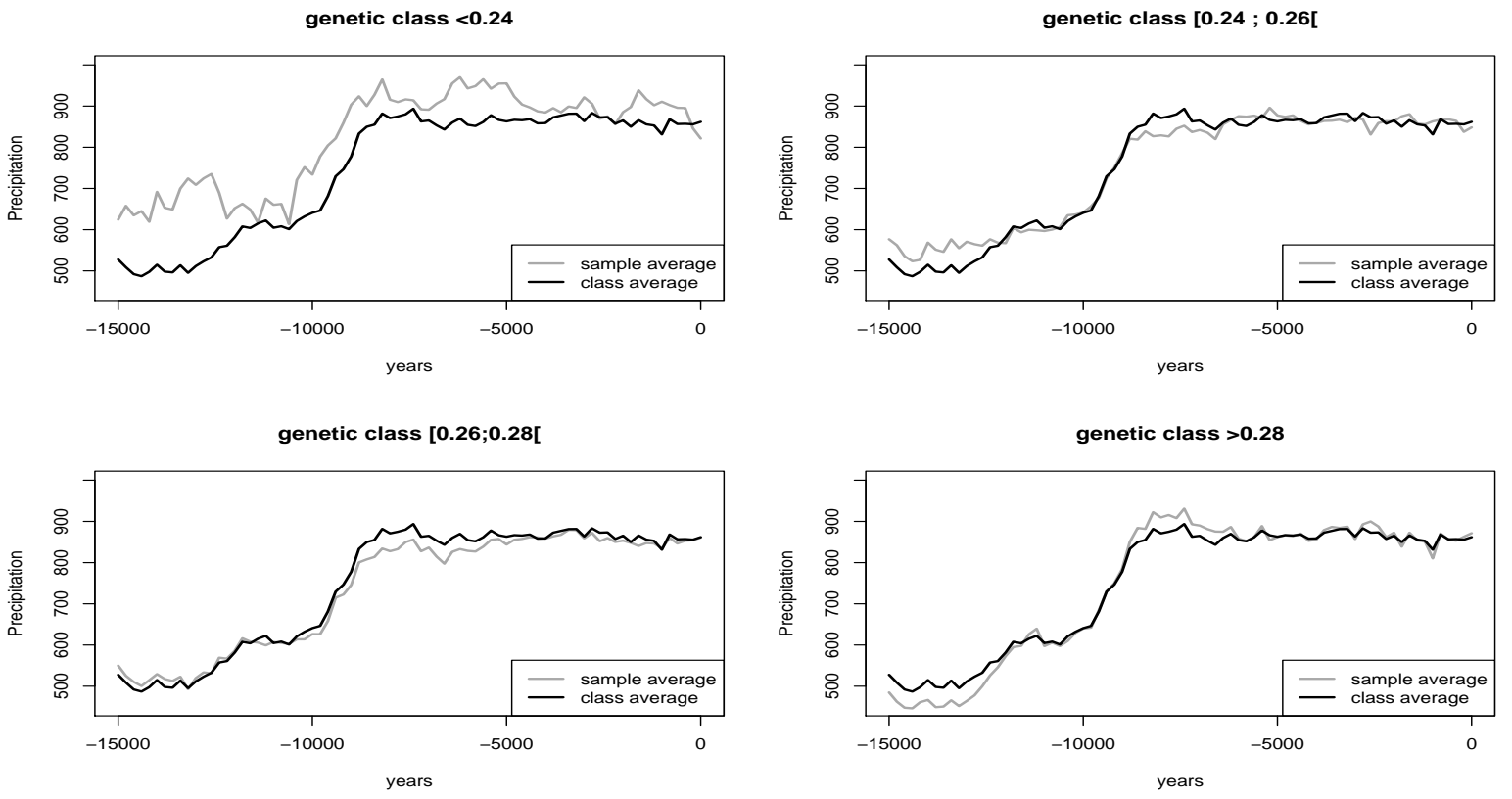

Figure 8. Pattern of precipitation curves according to the range of the predicted response.

1 To give a better understanding of the regression model we divide the predicted 
1

response range into 4 classes: less than 0.24, ]0.24;0.26], ]0.26;0.28] and greater than 0.28. Figures 7 and 8 show the shapes of temperature and precipitation curves for each class. When low $(<0.24)$ diversity is predicted, temperature curves are globally higher than the averaged temperature curves on all the sample. As the predicted diversity becomes higher the gap between the two periods before 7.5 Kyears and after 7.5 Kyears gets more pronounced. This effect is less evident for precipitation, low diversity is predicted when the precipitation is higher on the first period than the averaged precipitation curves on all the sample. When the predicted diversity is higher than 0.24 there seems to be no effect of precipitation on its the level.

When the change of climate during the Holocene (12 Kyears to present) is significant the diversity is higher. This mostly concerns northern and western Europe. This is coherent with previous studies [2]. After 12 Kyears and throughout the Holocene the climate was no longer uniform all over Europe. The largest mismatch between NW and SE Europe occurred around 9 Kyears and 5 Kyears. By 5 Kyears, all deciduous tree taxa (such as beech) were outside their glacial refugia.

\section{Conclusion}

The classical linear functional model has been extended in a straightforward manner to the case of two functional predictors with an interaction term, and with spatially correlated residuals. Such a model applied to complex paleoecological and biodiversity data emphasizes an interesting relationship between climate change and genetic diversity: diversity is higher when the change in climate (mostly temperature) during the Holocene (12 Kyears to present) was sizeable and lower when temperature and precipitation are both globally higher over the whole period. This model may be improved in several ways. The spatial effect may be handled in other ways, by means of a mixed structure or with other kinds of correlation matrix structure. In this first attempt we have neglected the random structure and the correlation of the predic- 
1 tors. Taking into account these two characteristics should give a better way 2 to understand the real effect of climate on biodiversity. 
1

3

5

\section{References}

[1] H. Cardot, F. Ferraty, P. Sarda, Functional linear model, Statist. Probab. Lett. 45 (1999) 11-22.

[2] R. Cheddadi, A. Bar-Hen, Spatial gradient of temperature and potential vegetation feedback across Europe during the late Quaternary, Climate Dynamics (in press).

[3] B. Comps, D. Gömöry, J. Letouzey, B. Thiébaut, R.J. Petit, Diverging trends between heterozygosity and allelic richness during postglacial colonization in the European beech, Genetics 157(2001) 389-397.

[4] N. Cressie, Statistics for spatial data, Revised Edition, Wiley, New-York, 1993

[5] D. Dahl-Jensen, K. Mosegaard, N. Gundestrup, G.D. Clow, S.J. Johnsen, A.W. Hansen, N. Balling, Past Temperatures Directly from the Greenland Ice Sheet, Science 282 (1998) 268-271.

[6] J. Fan, J.T. Zhang, Two-step estimation of functional linear models with application to longitudinal data. J. R. Stat. Soc. Ser. B Stat. Methodol. 62 (2000) 303-322.

[7] J.J. Faraway, Regression analysis for a functional response, Technometrics 39 (1997) 254-261.

[8] J. Guiot, Methodology of the last climatic cycle reconstruction in France from pollen data, Palaeogeography Palaeoclimatology Palaeo-ecology 80 (1990) 49-69.

[9] X. Guyon, Statistique et économétrie, Ellipses Marketing, Paris, 2001

[10] H.G. Müller, U. Stadtmüller, Generalized functional linear models, Ann. Stat. 33 (2005) 774-806

[11] J.O. Ramsay, B.W. Silverman, Functional Data Analysis, Springer, NewYork, 1997

[12] J. Seierstad, A. Nesje, S.O. Dahl, J.R. Simonsen, Holocene glacier fluctuations of Grovabreen and Holocene snow-avalanche activity reconstructed from lake sediments in Groningstolsvatnet, western Norway, The Holocene 12:2 (2002) 211-222. 